\title{
Next generation massively parallel sequencing of targeted exomes to identify genetic mutations in primary ciliary dyskinesia: Implications for application to clinical testing
}

\author{
Jonathan S. Berg, MD, PhD $D^{1,2}$, James P. Evans, $M D$, PhD ${ }^{1,2}$, Margaret W. Leigh, $M D^{3}$, \\ Heymut Omran, MD ${ }^{4}$, Chris Bizon, PhD ${ }^{5}$, Ketan Mane, $P h D^{5}$, Michael R. Knowles, $M D^{2}$, \\ Karen E. Weck, $M D^{1,6}$, and Maimoona A. Zariwala, $P h D^{6}$
}

\begin{abstract}
Purpose: Advances in genetic sequencing technology have the potential to enhance testing for genes associated with genetically heterogeneous clinical syndromes, such as primary ciliary dyskinesia. The objective of this study was to investigate the performance characteristics of exoncapture technology coupled with massively parallel sequencing for clinical diagnostic evaluation. Methods: We performed a pilot study of four individuals with a variety of previously identified primary ciliary dyskinesia mutations. We designed a custom array (NimbleGen) to capture 2089 exons from 79 genes associated with primary ciliary dyskinesia or ciliary function and sequenced the enriched material using the GS FLX Titanium (Roche 454) platform. Bioinformatics analysis was performed in a blinded fashion in an attempt to detect the previously identified mutations and validate the process. Results: Three of three substitution mutations and one of three small insertion/deletion mutations were readily identified using this methodology. One small insertion mutation was clearly observed after adjusting the bioinformatics handling of previously described SNPs. This process failed to detect two known mutations: one single-nucleotide insertion and a whole-exon deletion. Additional retrospective bioinformatics analysis revealed strong sequence-based evidence for the insertion but failed to detect the whole-exon deletion. Numerous other variants were also detected, which may represent potential genetic modifiers of the primary ciliary dyskinesia phenotype. Conclusions: We conclude that massively parallel sequencing has considerable potential for both research and clinical diagnostics, but further development is required before widespread adoption in a clinical setting. Genet Med 2011:13(3):218-229.
\end{abstract}

Key Words: next-generation sequencing, exon-capture, molecular diagnostic testing, primary ciliary dyskinesia, clinical genetics

From the Departments of ${ }^{1}$ Genetics, ${ }^{2}$ Medicine, and ${ }^{3}$ Pediatrics, the University of North Carolina Chapel Hill, Chapel Hill, North Carolina; ${ }^{4}$ Department of Paediatrics and Adolescent Medicine, University Hospital, Freiburg, Germany; ${ }^{5}$ The Renaissance Computing Institute, the University of North Carolina Chapel Hill; and ${ }^{6}$ Department of Pathology and Laboratory Medicine, the University of North Carolina Chapel Hill, Chapel Hill, North Carolina.

Jonathan S. Berg, MD, PhD, Department of Genetics, The University of North Carolina at Chapel Hill, Room 5092 Genetics Medicine Building, CB\# 7264, Chapel Hill, NC 27599-7264. E-mail: jsberg@med.unc.edu.

Disclosure: Karen E. Weck is a consultant for Roche Diagnostics. The other authors declare no conflict of interest.

Supplemental digital content is available for this article. Direct URL citations appear in the printed text and are provided in the HTML and PDF versions of this article on the journal's Web site (www.geneticsinmedicine.org).

Submitted for publication July 23, 2010

Accepted for publication October 26, 2010.

Published online ahead of print January 25, 2011.

DOI: $10.1097 /$ GIM.0b013e318203cff2
P rimary ciliary dyskinesia (PCD) is an autosomal recessive disorder involving abnormalities of motile cilia, resulting in a range of manifestations including situs inversus, neonatal respiratory distress at full-term birth, recurrent otitis media, chronic sinusitis, chronic bronchitis that may result in bronchiectasis, and male infertility. ${ }^{1-3}$ The disorder is genetically heterogeneous, rendering molecular diagnosis challenging given that mutations in nine different genes (DNAH5, DNAH11, DNAI1, DNAI2, KTU, LRRC50, RSPH9, RSPH4A, and TX$N D C 3$ ) account for only approximately $1 / 3$ of PCD cases. ${ }^{4}$ DNAH5 and DNAII account for the majority of known mutations, and the other genes each account for a small number of the remaining cases. ${ }^{5,6}$ Electron microscopy can reveal the presence of defective dynein arms or other axonemal components, and immunohistochemistry can suggest the loss of specific proteins, ${ }^{4}$ but in most cases, it is impossible to distinguish between patients with different genetic etiologies. Thus, from a diagnostic standpoint, it would be advantageous to engage in multiplex testing of multiple genes for causative mutations. Such an approach could also be readily adapted for gene discovery because the known involvement of ciliary genes in PCD suggests numerous "candidate genes," which are likely to play a role in cases of PCD without identifiable mutations.

Recent advancements in massively parallel sequencing (socalled "next-generation sequencing") are revolutionizing genetic research ${ }^{7-12}$ and demonstrate potential in clinical diagnostics. ${ }^{8,10,11,13-17}$ Because of the genetic heterogeneity in PCD, we investigated the performance characteristics of NimbleGen-targeted exon capture followed by massively parallel sequencing using Roche 454 technology 18 for detection of genetic variants in known and candidate PCD genes. We envision this technology as a hybrid platform capable of being used in the clinical diagnostic setting and in the research setting for those patients with no mutations in known PCD genes.

In this pilot study, we analyzed four patients with PCD in whom disease-causing mutations (three substitution mutations, three small insertion/deletion mutations, and one whole-exon deletion) were known. One of the patients had only one mutation identified previously, and we hoped to detect a second deleterious mutation. We expected a high detection rate for nonsense, small insertion/deletion, and missense mutations that were previously identified in the patient samples, but we recognized that this approach might fail to detect whole-exon deletions. We anticipated the discovery of "variants of uncertain significance" and "false positive" results, and we were interested in exploring the reasons for "false negative" results. The systematic analysis and troubleshooting of such results is a necessary prerequisite to the implementation of robust genomic analysis in the clinical arena. 


\section{MATERIALS AND METHODS}

\section{Patients and family members}

The patients were enrolled in a study of clinical and molecular aspects of $\mathrm{PCD}^{1}$ approved by the Institutional Review Board at the University of North Carolina at Chapel Hill (study no. 05-2979). DNA was prepared by salt extraction from lymphoblastoid cell lines (patients 475 and 1205) or blood samples (patients 998 and 1072). DNA quality was measured by ultraviolet spectrometry (A260/280 ratios between 1.84 and 1.89 for all samples) and gel electrophoresis. The sample from patient 475 had a small amount of degradation but was deemed acceptable for use. None of the patients came from consanguineous families.

\section{Exon-capture design, enrichment, and sequencing}

We designed a custom oligonucleotide microarray (NimbleGen) to capture 2089 exons of 79 genes known to be associated with PCD or candidate genes based on function/expression in cilia/flagella of human or model organisms (Tables, Supplemental Digital Contents 1 and 2, http://inks.lww.com/GIM/A129 and http://inks.lww.com/GIM/A131). The final targeted region included 510,558 base pairs (bp; 95.6\%) with an offset of 0 bases or 520,838 base pairs $(97.5 \%)$ with an offset of 100 bases. DNA samples from each of the patients were sent to Roche and subjected to capture and 454 sequencing according to standard operating procedures. Roche performed mapping, alignment, and variant detection against the human National Center for Biotechnology Information (NCBI). Build 36 reference genome using the GS Reference Mapper software package. ${ }^{19}$ Samples from patients 475 and 998 underwent an additional round of sequencing due to concerns about read length in the initial sequencing runs.

\section{Variant analysis}

One investigator, blinded to the identity of the previously determined disease-causing mutations, analyzed all variants within the targeted region for the four patients. The HYDIN gene on chromosome 16q22 was excluded from our analysis due to the existence of a paralog mapping to chromosome 1q21 that is not accounted for in the NCBI 36 reference sequence. Variants identified by GS Reference Mapper are annotated with genomic coordinates, reference nucleotide, variant nucleotide, number of reads containing the variant nucleotide, and percentage of reads containing the variant nucleotide. Additional annotations (if applicable) include the reference amino acid, variant amino acid, reading frame, gene name, and overlap with a known single nucleotide polymorphism (SNP) from dbSNP 130. For identification of possible splice site mutations, we calculated the distance of each variant to the nearest intron-exon junction and then selected variants occurring within the last two nucleotides of an exon and first five nucleotides of the donor intron, or within the last five nucleotides of the intron and the first nucleotide of the acceptor exon. Further characterization of missense mutations was performed on selected variants using evolutionary conservation and prediction of pathogenicity with PolyPhen. ${ }^{20,21}$ PCR amplification and follow-up Sanger sequencing of newly identified candidate mutations were performed.

\section{Statistical analysis}

Tables of variants were organized using Microsoft Excel. Statistical analysis and graphing were performed using GraphPad Prism (version 5.0b).

\section{RESULTS}

\section{Previous characterization of disease-causing mutations in patients with PCD}

Our group has performed extensive analysis of selected genes in patients with PCD and family members. For this study, four previously characterized patients were selected based on qualitatively different types of mutations in three PCD genes (summarized in Table 1).

- Patient 475: Sanger sequencing of DNAH11, WDR63, RSHL1, RSPH3, RSPH4A, DNAH5, and selected exons of DNAII revealed compound heterozygous mutations in DNAH5: a missense mutation in the last base of an exon leading to a splicing defect and a 21-bp deletion causing an in-frame deletion of seven amino acid residues.

- Patient 998: Sanger sequencing of the entire coding regions of DNAH11, DNAH5, and DNAII identified only one mutation in DNAH11, a 4-bp deletion leading to a frameshift.

- Patient 1072: Sanger sequencing of DNAH5 and DNAII revealed compound heterozygous nonsense mutations in DNAII resulting in premature truncation.

- Patient 1205: Analysis of DNAH11 and selected exons of $D N A H 5$ revealed compound heterozygous mutations in DNAH5: one single-base insertion leading to a frameshift detected by Sanger sequencing and a deletion of exon 62 detected by multiplex ligation-dependent probe amplification (MLPA).

Table 1 Patient characteristics and known mutations

\begin{tabular}{|c|c|c|c|c|c|c|}
\hline $\begin{array}{l}\text { Patient } \\
\text { (age, sex) }\end{array}$ & $\begin{array}{l}\text { Clinical } \\
\text { presentation }\end{array}$ & $\mathrm{nNO}$ & EM features & Gene & Mutation 1 & Mutation 2 \\
\hline 475 (14 yr, F) & $\begin{array}{l}\text { NRD, SI, Bx, } \\
\text { Sx, OM }\end{array}$ & $10.7 \mathrm{~nL} / \mathrm{min}$ & ODA defect & DNAH5 & $\begin{array}{l}\text { c.6249G }>\text { A (p.M2083I, } \\
\text { splice site) }\end{array}$ & $\begin{array}{l}\text { c.7468_7488 del } \\
\text { (p.2490_2496 del } \\
\text { WSAGAAL) }\end{array}$ \\
\hline 998 (29 yr, M) & $\mathrm{Bx}, \mathrm{Sx}, \mathrm{OM}$ & $70.4 \mathrm{~nL} / \mathrm{min}$ & $\begin{array}{l}\text { Normal dynein arms and } \\
\text { central apparatus }\end{array}$ & DNAH11 & $\begin{array}{c}\text { c.9113_9116delAAGA } \\
\text { (p.K3038TfsX13) }\end{array}$ & Not identified \\
\hline $1072(12 \mathrm{yr}, \mathrm{M})$ & NRD, Sx, OM & $10.1 \mathrm{~nL} / \mathrm{min}$ & ODA defect & DNAII & c. $1212 \mathrm{~T}>\mathrm{G}(\mathrm{p} . \mathrm{Y} 404 \mathrm{X})$ & c.1644G $>$ A (p.W548X) \\
\hline $1205(16 \mathrm{yr}, \mathrm{F})$ & $\begin{array}{l}\text { NRD, SI, Bx, } \\
\text { Sx, OM }\end{array}$ & $18.9 \mathrm{~nL} / \mathrm{min}$ & ODA defect & DNAH5 & $\begin{array}{l}\text { Del exon } 62 \text { by MLPA } \\
\quad \text { (junctions not known) }\end{array}$ & $\begin{array}{l}\text { c.13458_59insT } \\
\text { (p.N4487fsX1) }\end{array}$ \\
\hline
\end{tabular}

nNO, nasal nitric oxide (normal range: $376 \pm 124 \mathrm{~nL} / \mathrm{min}$, mean $\pm \mathrm{SD}$ ); EM, electron microscopy; NRD, neonatal respiratory distress; SI, situs inversus; Bx, bronchiectasis; Sx, sinusitis; OM, frequent otitis media; ODA, outer dynein arm; MLPA, multiplex ligation-dependent probe amplification. 
Exon capture, high-throughput sequencing, mapping, and variant identification

Patient DNA was enriched for 2089 exons from 79 known or predicted PCD genes, using a custom NimbleGen oligonucleotide capture array. Roche GS FLX Titanium massively parallel sequencing of the enriched material yielded 760,738-1,174,723 high-quality reads encompassing 249,757,548-363,712,243 high-quality bases per patient (see Tables, Supplemental Digital Contents 3 and 4, http://links.lww.com/GIM/A132 and http://links.lww.com/GIM/A133). After mapping, $>99.8 \%$ of the targeted region was covered in each patient, and $>97.7 \%$ of the targeted region was covered at $>10$-fold depth. Weighted unique mean depth of coverage for the targeted region was $39 x-89.2 x$. Thus, the coverage should have been adequate to reliably detect germline variants within the majority of targeted regions. There was an average of 538 variants per patient within the 510,558-bp-targeted exome region $(\sim 1$ variant per $\mathrm{kb}$ of targeted genome). Roche considers variants observed in 15$85 \%$ of unique reads to be heterozygous, and the GS Reference Mapper algorithm matches variants to dbSNP version 130 . Using these criteria, there were 32-53 novel variants detected per patient sample (Table 2).

\section{Identification of candidate disease-causing mutations}

As a platform such as this one could eventually be used in a clinical diagnostic setting, we attempted to develop an analytic process compatible with the workflow of a typical molecular diagnostic laboratory, in which variants meeting the following criteria would be considered potentially causative: (i) detected in $>15 \%$ of reads, (ii) not overlapping with an annotated SNP, (iii) predicted to be truncating (premature stop/frameshift/splice-site disruption) or altering an amino acid, and (iv) presence of two mutations in one gene, consistent with autosomal recessive inheritance (summarized in Table 2). Although such a scheme would miss rare mutations in regulatory regions or splice enhancer sequences, it would be expected to identify the majority of clinically relevant mutations. Variants identified in the initial workflow are further detailed in Table 3 .

\section{Patient 475}

The initial blinded approach identified both of the previously characterized disease-causing mutations in DNAH5. The heterozygous 21-bp deletion in the DNAH5 gene was present in $57 \%$ of the reads. The second mutation in DNAH5 was observed in $52 \%$ of sequence reads and consisted of a substitution that changes a methionine residue to leucine and involves the last nucleotide of the exon, thus disrupting a canonical splice donor sequence.

This individual had three other genes (RSPH4A, DNAH10, and $L R R C 50$ ) each with two novel variants. Both $R S P H 4 A$ and $L R R C 50$ have been associated with PCD, ${ }^{22,23}$ whereas DNAH10 is an inner arm dynein heavy chain ${ }^{24}$ but has not been extensively analyzed in patients with PCD. The two RSPH4A variants were both single-base insertions that would cause a frameshift but were observed in a low percentage of reads. Interestingly, previous analysis of the RSPH4A gene by Sanger sequencing had identified three common polymorphisms in this patient but no evidence for the putative novel insertions, indicating that they are likely false-positive results of 454 sequencing. One of the variants in DNAH10 changed a highly conserved arginine to tryptophan. The other variant altered an evolutionarily nonconserved histidine to tyrosine and was also observed in one other patient, suggesting that it is likely benign. The two variants in

Table 2 Candidate mutations identified through an initial blinded analysis of variant data

\begin{tabular}{|c|c|c|c|c|}
\hline & Patient 475 & Patient 998 & Patient 1072 & Patient 1205 \\
\hline Total high-confidence variants & 18,042 & 19,877 & 12,332 & 10,560 \\
\hline In region $(-H Y D I N)$ & 521 & 435 & 449 & 456 \\
\hline$>15 \%$ of reads & 515 & 423 & 443 & 435 \\
\hline Novel & 51 & 31 & 28 & 49 \\
\hline Intronic & 29 & 21 & 16 & 31 \\
\hline Synonymous & 4 & 2 & 3 & 5 \\
\hline Missense & 10 & 7 & 7 & 8 \\
\hline Nonsense & 0 & 0 & 2 & 0 \\
\hline Small ins/del & 7 & 1 & 0 & 4 \\
\hline Near splice site & 1 & 0 & 0 & 4 \\
\hline Candidate mutations & 17 & 8 & 9 & 16 \\
\hline
\end{tabular}

Genes with two candidate mutations

DNAH5

RSPH4A ${ }^{a}$

$D N A H 9^{a}$

\footnotetext{
${ }^{a}$ False-positive insertion mutation.

${ }^{b}$ Variants of uncertain significance.

${ }^{c}$ Determined to be in cis by Sanger sequencing

${ }^{d}$ Likely sequencing artifact.
} 
Table 3 Summary of candidate mutations identified or missed by exon capture and massively parallel sequencing

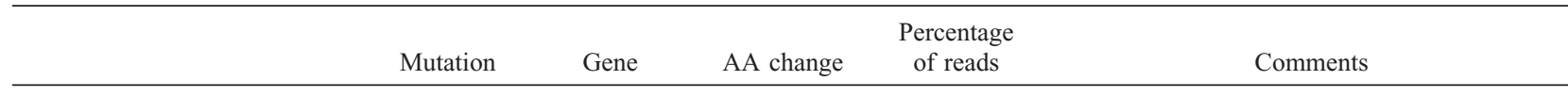

\section{Patient 475}

chr5: $13,863,923-13,863,313$

chr5: $13,883,135$

Del $21 \mathrm{bp}$

DNAH5

$\mathrm{C}>\mathrm{T}$

DNAH5

del WSAGAAL

$\mathrm{M}-\mathrm{I}$

\section{Possible compound heterozygous variants}

$\begin{array}{llll}\text { chr6: } 117,045,024 & \text { Ins T } & \text { RSPH4A } & \text { Q-L, fs } \\ \text { chr6: } 117,058,303 & \text { Ins C } & \text { RSPH4A } & \text { Y-L, fs } \\ \text { chr12: } 122863856 & \mathrm{C}>\mathrm{T} & \text { DNAH10 } & \mathrm{R}-\mathrm{W} \\ \operatorname{ch} 12: 122864030 & \mathrm{C}>\mathrm{T} & \text { DNAH10 } & \mathrm{H}-\mathrm{Y} \\ \operatorname{ch} 16: 82,761,275-82,761,277 & \text { CAC }>\text { A } & \text { LRRC50 } & \text { PP-HT, fs } \\ \text { chr16: } 82,761,284-82,761,286 & \text { CGC }>\mathrm{G} & \text { LRRC50 } & \text { PP-RT, fs }\end{array}$

\section{Heterozygous variants}

chr1: 223335050
chr2: 84865382
chr5: 35763614
chr7: 21907348
chr11: 102596607
chr14: 101519552
chr19: 53492145
chr21: 42769212

$\mathrm{A}>\mathrm{T}$

DNAH14

H-L

Ins $\mathrm{T}$

DNAH6

$\mathrm{R}-\mathrm{R}$, fs

$\mathrm{G}>\mathrm{A}$

SPEF2

A-T

$\mathrm{A}>\mathrm{G}$

DNAHI1

$\mathrm{K}-\mathrm{R}$

$\mathrm{G}>\mathrm{A}$

DYNC2H1 E-K

Ins A

DYNC1H1 V-V, fs

$\mathrm{G}>\mathrm{A}$

CCDC114 S-L

$\mathrm{C}>\mathrm{T}$

RSPHI

G-R

$\mathrm{T}>\mathrm{C}$

$R P G R$

Q-R

Disease-causing mutations

\section{Patient 998}

chr7: 2177919-21779922

Del AAGA

DNAH11

KD-TL, fs

Possible compound heterozygous variants

chr17: 11491146

chr17: 11549161

chr12: 109829602

chr12: 122864030

chr17: 7677205

chr21: 42769212

chr2: 84778334

chr16: 20882241

\section{Patient 1072}

chr9: 34496773

chr9: 34504466
$\mathrm{G}>\mathrm{A}$

DNAH9

$\mathrm{R}-\mathrm{Q}$

Ins $\mathrm{T}$

DNAH9

Q-L, fs

\section{Heterozygous variants}

$\begin{array}{lll}\mathrm{G}>\mathrm{T} & \text { CCDC63 } & \mathrm{R}-\mathrm{L} \\ \mathrm{C}>\mathrm{T} & \text { DNAH10 } & \mathrm{H}-\mathrm{Y} \\ \mathrm{T}>\mathrm{A} & \text { DNAH2 } & \mathrm{V}-\mathrm{D} \\ & & \\ \mathrm{C}>\mathrm{T} & \text { RSPH1 } & \mathrm{G}-\mathrm{R}\end{array}$

\section{Homozygous variants}

$\mathrm{T}>\mathrm{C}$

DNAH6

$\mathrm{C}>\mathrm{T}$

DNAH3

$\mathrm{V}-\mathrm{A}$

$\mathrm{R}-\mathrm{Q}$

Disease-causing mutations

$\begin{array}{lll}\mathrm{T}>\mathrm{G} & \text { DNAII } & \text { Y-Stop } \\ \mathrm{G}>\mathrm{A} & \text { DNAII } & \text { W-Stop }\end{array}$

57

52

17

24

52

50

17

28

53

16

34

57

58

20

48

44

55

46

18

44

51

61

100

100

53

44
Deletion of 7 amino acids

Last nucleotide of exon, likely splice donor site mutation

False positive, not detected by Sanger sequencing False positive, not detected by Sanger sequencing Highly conserved except $\mathrm{G}$ in canine Poorly conserved; Also present in patient 998 Located within simple tandem repeat, likely artifact Located within simple tandem repeat, likely artifact

Highly conserved

False positive, not detected by Sanger sequencing Poorly conserved AA

Not highly conserved ( $\mathrm{R}$ in mouse)

Invariant amino acid

Possible frameshift but likely false positive

Not highly conserved ( $\mathrm{L}$ in mouse)

Poorly conserved; Also present in patient 998

Poorly conserved

Bioinformatics false negative: matched with rs72657376

Poorly conserved ( $\mathrm{Q}$ is common); confirmed by Sanger sequencing

False positive, not detected by Sanger sequencing

Poorly conserved

Poorly conserved; also present in patient 475

Invariant amino acid; confirmed by Sanger sequencing

Poorly conserved; also present in patient 475

Poorly conserved; also present in patient 1205

Poorly conserved ( $\mathrm{Q}$ is common)

Nonsense mutation

Nonsense mutation

(Continued) 
Table 3 Continued

\section{Mutation Percentage}

\begin{tabular}{|c|c|c|c|}
\hline chr6: 39005350 & $\mathrm{~A}>\mathrm{G}$ & DNAH8 & $\mathrm{H}-\mathrm{R}$ \\
\hline chr6: 39007668 & $\mathrm{C}>\mathrm{T}$ & DNAH8 & $\mathrm{T}-\mathrm{M}$ \\
\hline
\end{tabular}

Poorly conserved ( $\mathrm{R}$ is common); Confirmed by Sanger sequencing but in cis with other DNAH8 variant

Highly conserved except S in lizard; Confirmed by Sanger sequencing but in cis with other DNAH8 variant

\section{Heterozygous variants}

\begin{tabular}{|c|c|c|c|c|c|}
\hline chr1: 36323224 & $\mathrm{C}>\mathrm{T}$ & $T E K T 2$ & $\mathrm{R}-\mathrm{C}$ & 55 & $\begin{array}{l}\text { Invariant amino acid; Confirmed by Sanger } \\
\text { sequencing }\end{array}$ \\
\hline chr2: 196446610 & $\mathrm{~T}>\mathrm{C}$ & $D N A H 7$ & $\mathrm{~T}-\mathrm{A}$ & 63 & Invariant amino acid \\
\hline chr16: 82746850 & $\mathrm{G}>\mathrm{A}$ & $L R R C 50$ & $\mathrm{D}-\mathrm{N}$ & 50 & $\begin{array}{l}\text { Not highly conserved ( } \mathrm{N} \text { in chicken); Confirmed } \\
\text { by Sanger sequencing }\end{array}$ \\
\hline chr17: 7641273 & $\mathrm{~A}>\mathrm{G}$ & $D N A H 2$ & $\mathrm{I}-\mathrm{V}$ & 35 & Highly conserved; conservative substitution \\
\hline chr17: 11495120 & $\mathrm{G}>\mathrm{A}$ & $D N A H 9$ & $\mathrm{~V}-\mathrm{M}$ & 50 & Invariant amino acid; conservative substitution \\
\hline Patient 1205 & \multicolumn{5}{|c|}{ Disease-causing mutations } \\
\hline Exon 62 deletion & $\mathrm{N} / \mathrm{A}$ & $D N A H 5$ & $\mathrm{~N} / \mathrm{A}$ & $\mathrm{X}$ & Not identified \\
\hline chr5: 13754432 & Ins A & DNAH5 & N-Stop, fs & $\mathrm{X}$ & Not identified; evidence from sequence alignment \\
\hline & \multicolumn{5}{|c|}{ Possible compound heterozygous variants } \\
\hline chr1: 118349567 & $\mathrm{G}>\mathrm{T}$ & $S P A G 17$ & $\mathrm{~T}-\mathrm{N}$ & 41 & $\begin{array}{l}\text { Predicted damaging; Confirmed by Sanger } \\
\text { sequencing }\end{array}$ \\
\hline \multirow[t]{2}{*}{ chr1: 118425275} & $\mathrm{C}>\mathrm{T}$ & $S P A G 17$ & $\mathrm{M}-\mathrm{I}$ & 45 & $\begin{array}{l}\text { Not highly conserved, predicted benign; } \\
\text { Confirmed by Sanger sequencing }\end{array}$ \\
\hline & \multicolumn{5}{|c|}{ Heterozygous variants } \\
\hline chr2: 27530434 & Ins $\mathrm{C}$ & IFT172 & $\mathrm{A}-\mathrm{P}, \mathrm{fs}$ & 16 & False positive, not detected by Sanger sequencing \\
\hline chr2: 214502993 & $\mathrm{G}>\mathrm{A}$ & $S P A G 16$ & $\mathrm{D}-\mathrm{N}$ & 44 & Poorly conserved ( $\mathrm{N}$ in dog) \\
\hline chr5: 35736480 & Ins $\mathrm{A}$ & $S P E F 2$ & $\mathrm{~A}-\mathrm{D}, \mathrm{fs}$ & 22 & False positive, not detected by Sanger sequencing \\
\hline chr6: 38993126 & Ins A & $D N A H 8$ & $\mathrm{~S}-\mathrm{Y}, \mathrm{fs}$ & 15 & False positive, not detected by Sanger sequencing \\
\hline chr11: 102532339 & $\mathrm{C}>\mathrm{T}$ & $\mathrm{DYNC} 2 \mathrm{H1}$ & $\mathrm{R}-\mathrm{W}$ & 56 & Invariant amino acid \\
\hline chr12: 109796040 & Ins $\mathrm{A}$ & $C C D C 63$ & $\mathrm{M}-\mathrm{I}, \mathrm{fs}$ & 15 & Possible frameshift but likely false positive \\
\hline chr12: 122854217 & $\mathrm{G}>\mathrm{A}$ & DNAH10 & $\mathrm{V}-\mathrm{I}$ & 53 & Highly conserved; conservative substitution \\
\hline chr16: 21030816 & $\mathrm{G}>\mathrm{A}$ & $D N A H 3$ & $\mathrm{~T}-\mathrm{M}$ & 55 & Not highly conserved ( $\mathrm{M}$ in Rhesus) \\
\hline chr16: 82746855 & Ins $\mathrm{A}$ & $L R R C 50$ & $\mathrm{~L}-\mathrm{L}, \mathrm{fs}$ & 18 & False positive, not detected by Sanger sequencing \\
\hline $\operatorname{chr17:} 7603162$ & $\mathrm{C}>\mathrm{T}$ & $D N A H 2$ & $\mathrm{P}-\mathrm{S}$ & 55 & Not highly conserved ( $\mathrm{S}$ in elephant) \\
\hline chr17: 73988359 & $\mathrm{~A}>\mathrm{T}$ & DNAH17 & $\mathrm{N} / \mathrm{A}$ & 50 & $\begin{array}{l}\text { Possible splice donor mutation (intron }+2 \\
\text { position) }\end{array}$ \\
\hline
\end{tabular}

\section{Homozygous variants}

LRRC50 were both observed in a low percentage of reads and were located in a short region of low complexity sequence (CCCCACCACCCCCGCCACC) within a simple tandem repeat, suggesting that they are sequencing artifacts.
Heterozygous variants of uncertain significance were identified in nine other genes (DNAH14, DNAH6, SPEF2, DNAH11, DYNC2H1, DYNC1H1, CCDC114, RSPH1, and $R P G R)$. One of these (DNAH6) was an insertion detected in 


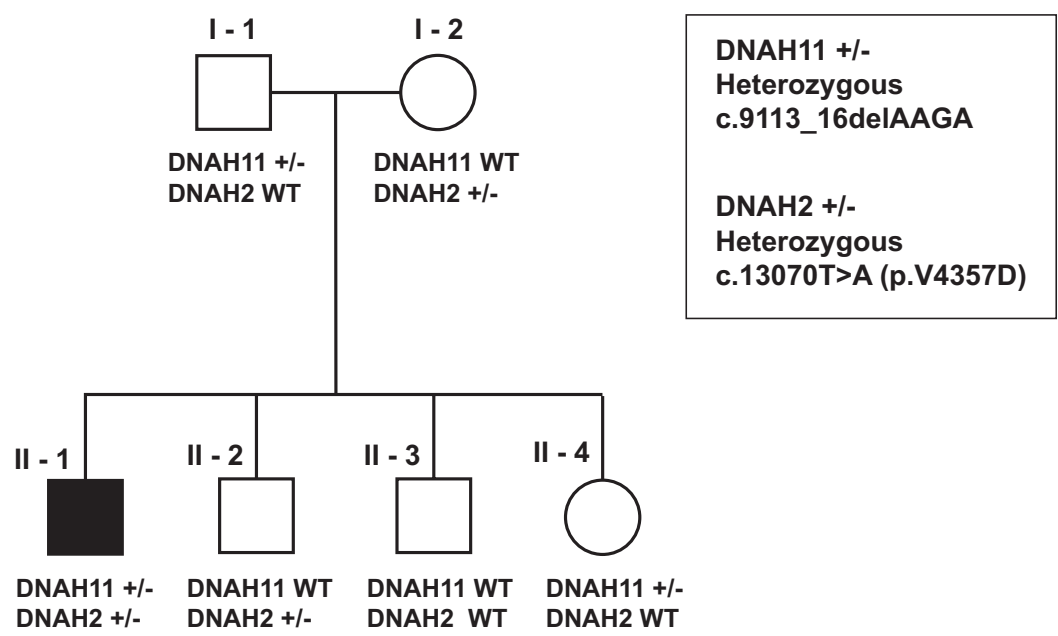

Fig. 1. Family segregation analysis supports the possibility of digenic inheritance of DNAH11 and DNAH2 mutations in patient $998(\square)$. A patient with a phenotype consistent with PCD (II-1) was previously found to have a single mutation in DNAH11, which encodes a component of the outer dynein arm. Targeted exome sequencing found no additional possible disease-causing mutations in DNAH11 but identified a heterozygous missense mutation of a highly conserved residue of $D N A H 2$, which encodes a component of the inner dynein arm. Family segregation analysis revealed that the DNAH11 mutation was inherited from the patient's father (I-1), whereas the DNAH2 mutation was inherited from the patient's mother (I-2). Furthermore, the three unaffected siblings were heterozygous for only the DNAH2 allele (brother II-2), heterozygous for only the DNAH11 allele (sister II-4), or wild type for both alleles (brother II-3).

$16 \%$ of sequence reads that was not detected on subsequent Sanger sequencing.

\section{Patient 998}

This patient was the only one in whom only a single diseasecausing mutation had been previously identified-a 4-bp deletion in the DNAH11 gene associated with PCD and normal axonemal ultrastructure. ${ }^{25}$ It was, therefore, hoped that two deleterious mutations would be identified in DNAH11 or in another known or candidate PCD gene.

The initial blinded analysis failed to identify the known 4-bp deletion mutation in DNAH11 previously detected by Sanger sequencing. The only gene identified as having possible heterozygous variants was $D N A H 9$, a candidate gene for PCD. ${ }^{26}$ One $D N A H 9$ variant was a single-base insertion observed in $18 \%$ of sequence reads, but it was not detected on Sanger sequencing. The other DNAH9 variant changes an arginine residue to glutamine, but glutamine is observed at this site in other species, suggesting that the variant is benign. Together, these results argue strongly against $D N A H 9$ as the cause of PCD in this individual.

Two dynein heavy-chain genes (DNAH3 and DNAH6) demonstrated novel homozygous variants. The $D N A H 3$ variant changes an arginine residue to glutamine, but several species have a glutamine at this position indicating that it is unlikely to be deleterious. The DNAH6 variant changes valine to alanine but is again poorly conserved; in addition, this variant was also detected in the homozygous state in patient 1205 . Therefore, both of these homozygous variants most likely represent benign SNPs.

Because some known disease-causing mutations are present in the dbSNP database, ${ }^{27}$ we reconsidered all coding variants in this individual, regardless of whether they were annotated as overlapping with a previously described SNP. In doing so, we recognized the previously detected 4-bp frameshift deletion in DNAH11, clearly present in $53 \%$ of the reads. This variant had been annotated as overlapping with SNP rs72657376 and was, therefore, eliminated from consideration in the initial analytic scheme. This false negative represents a bioinformatics failure, because the use of dbSNP data as an analytic filter caused us initially to miss this mutation. Further analysis of DNAH11 revealed several other known SNPs but no nonsense or likely splice site mutations. Thus, after adjusting the analytic scheme (albeit in a post hoc fashion), we were ultimately able to identify the 4-bp deletion but no other likely disease-causing mutations in DNAH11.

Four additional genes (CCDC63, DNAH2, DNAH10, and RSPH1) were found to contain novel heterozygous missense variants. In $D N A H 10$ and $R S P H 1$, the affected amino acids were poorly conserved among other species, and both of the variants were present in patient 475 , suggesting that they are likely to be benign. The variant in the CCDC63 gene also affects a poorly conserved amino acid. However, we were intrigued by a novel heterozygous missense variant in DNAH2 (chr17: 7677205 $\mathrm{T}>\mathrm{A}$ ), altering a highly conserved valine residue to aspartic acid predicted by PolyPhen to be possibly damaging. This variant was not observed in any of the three other patients. We, therefore, considered whether the patient's phenotype could be due to digenic inheritance in this case (Fig. 1). Testing of family members revealed that the DNAH11 mutation was inherited from the patient's father, and the $D N A H 2$ variant was inherited from the patient's mother. Among the three unaffected siblings, the sister carries only the DNAH11 mutation, a brother carries only the $D N A H 2$ variant, and another brother is wild type at both loci. The familial segregation is consistent with the possibility of digenic inheritance, although further studies will be required to establish this with certainty.

\section{Patient 1072}

The initial blinded analysis revealed two heterozygous nonsense mutations in the DNAI1 gene, which has been previously associated with PCD. ${ }^{28}$ These were the precise disease-causing mutations that had previously been identified by Sanger sequencing. 
In addition, two novel heterozygous variants in the dynein heavy-chain gene DNAH8 were identified. One of the DNAH8 variants predicts a change of a histidine residue to arginine, which is observed at this position in numerous other species and was predicted by PolyPhen to be benign. The other DNAH8 variant changes a threonine residue to methionine and was predicted by PolyPhen to be probably damaging. Both variants were confirmed by Sanger sequencing but were found to have been inherited in cis from the mother, whereas the father was wild type at both positions. Thus, only one allele of DNAH8 is affected by these changes. This individual also had heterozygous missense mutations of highly conserved or invariant amino acids in several other dynein heavy-chain genes (DNAH2, DNAH7, and DNAH9) and Tektin-2 (TEKT2), which encodes a protein that is coassembled with ciliary and flagellar microtubules and may play a role in asthenozoospermia. ${ }^{29}$

\section{Patient 1205}

Neither deleterious mutation previously identified in this patient (a large exonic deletion and a single-nucleotide insertion in DNAH5) was detected by massively parallel sequencing in our established analytic scheme.

The initial blinded analysis identified only one gene, $S P A G 17$, as having two candidate variants. The first of these variants changes a methionine to isoleucine and was predicted by PolyPhen to be benign, whereas the second variant changes a threonine residue to asparagine and was predicted by PolyPhen to be damaging. The SPAG17 gene encodes an ortholog of Chlamydomonas PF6, a component of the flagellar central apparatus. ${ }^{30}$ Mutations of the Chlamydomonas ortholog cause flagellar paralysis, but phenotypes have not been reported in humans or mice. Sanger sequencing confirmed the presence of both variants, but their clinical significance is uncertain.

This individual had 11 other genes with a single heterozygous novel variant, including four dynein heavy-chain genes (DNAH2, DNAH3, DNAH8, and DNAH10) and a gene associated with intraflagellar transport (IFT172). In addition, the $D N A H 6$ variant that was detected in patient 998 was observed in $100 \%$ of the reads in this individual. Of the 11 novel heterozygous variants, five were single-base insertions that would be predicted to cause a frameshift, but all five were present in $<25 \%$ of the sequence reads. We selected four of these variants (IFT172, SPEF2, DNAH8, and LRRC50) for confirmation by Sanger sequencing, and none of them were confirmed. The clinical significance of the remaining heterozygous missense variants remains uncertain at this time.

Having failed to identify likely disease-causing mutations in this individual, we unblinded the analysis and attempted to find the two mutations in DNAH5 previously discovered through Sanger sequencing and MLPA. Given that exon capture is nonquantitative, we did not necessarily expect to find the exon deletion using this platform, and indeed, on examining sequence-based data and depth of coverage across all exons, we could not detect any evidence of the exon deletion (see Text and Figure, Supplemental Digital Content 5, http://links.lww.com/GIM/A134). However, we were surprised that the insertion mutation in DNAH5 (c.13458_59 insT) was not identified, even among low-quality variants that failed to meet the "high confidence" standards. This mutation has been observed in multiple families and may represent a founder mutation. ${ }^{5}$ The DNAH5 cDNA is transcribed from the minus strand, and the c.13458_59 insT mutation localizes to a stretch of 7 adenine (7A) nucleotides on the plus strand of the reference genomic sequence (chr5: 13,754,426-13,754,432). Thus, the c.13458_59 insT mutation should result in 8 adenines (8A) at this position.
On the basis of previous reports regarding the deficiency of 454 sequencing in regions containing short homopolymers, ${ }^{31}$ we hypothesized that the number of adenine residues in different reads at this position should follow a normal distribution around the "true" number. We, therefore, made local alignments of sequence reads (Fig. 2A) mapping to this location in all four patients, tabulated the number of adenine nucleotides in each read, and compared the proportion of reads containing $5,6,7,8,9$, or 10 reads between each patient (Fig. 2B). The sequence data for patients 475, 998, and 1072 were consistent with a homozygous 7A genotype, whereas the sequence data for patient 1205 suggested a heterozygous 7A/8A genotype (Fig. 2C), consistent with the prior Sanger sequencing results. Thus, the massively parallel sequencing data support the presence of a mutant $8 \mathrm{~A}$ allele at this position when analyzed in a post hoc manner, but the GS Reference Mapper variant detection algorithm did not identify it prospectively.

Raca et al. ${ }^{32}$ reported a similar problem with 454 sequencing in a pilot study of massively parallel sequencing for ocular birth defects. These authors reported an initial failure, also using the GS Reference Mapper software, to detect a single-base deletion within a stretch of 7 guanine nucleotides in the $P A X 2$ gene. However, they were able to demonstrate the presence of the deletion using two different software packages (CLC Genomic Workbench and NextGENe). We similarly used NextGENe to independently assemble the raw sequence reads and call variants, and indeed, the heterozygous insertion of adenine was recognized at this location (data not shown). However, this increased sensitivity came at the cost of greatly increasing the number of putative single-nucleotide insertions and deletions. For instance, using default settings, NextGENe called five or more deletion variants in the coding regions of several genes (observed in $>25 \%$ of reads and in some cases $\sim 50 \%$ of reads) that were not called by 454's suite and which occurred in the immediate context of homopolymer stretches, strongly suggesting that they are false positives.

\section{Analytical sensitivity and specificity of exon capture followed by massively parallel sequencing}

An important potential limitation of this approach is that it may be prone to both false-positive and false-negative results. This was clearly the case with the four patient samples we analyzed. In the course of evaluating novel variants, we used Sanger sequencing to follow-up 16 variants identified by 454 sequencing. All eight of the putative novel single-base insertion variants, observed in $15-24 \%$ of sequence reads, were determined to be false positives of 454 sequencing based on the negative results of confirmatory Sanger sequencing. In contrast, Sanger sequencing confirmed all eight novel missense variants, observed in $41-56 \%$ of sequence reads.

As we inspected the next-generation sequencing data as a whole, it became clear that a large number of putative novel small insertion/deletion variants were observed in fewer than $25 \%$ of the 454 sequence reads and that this might be a useful signal for flagging possible false-positive results. To further explore this phenomenon, we analyzed the percentage of sequence reads in which different types of variants were identified (Fig. 3). We noted that insertions and deletions were likely to be detected in a lower percentage of reads than single-base substitutions, which clustered near $50 \%$ and $100 \%$ of reads, as would be expected for heterozygous or homozygous variants, respectively. Putative "novel" (not annotated in dbSNP) insertion/ deletion variants were much more frequently observed in fewer than $25 \%$ of sequence reads compared with "known" (previously annotated in dbSNP) insertion/deletion variants or novel 

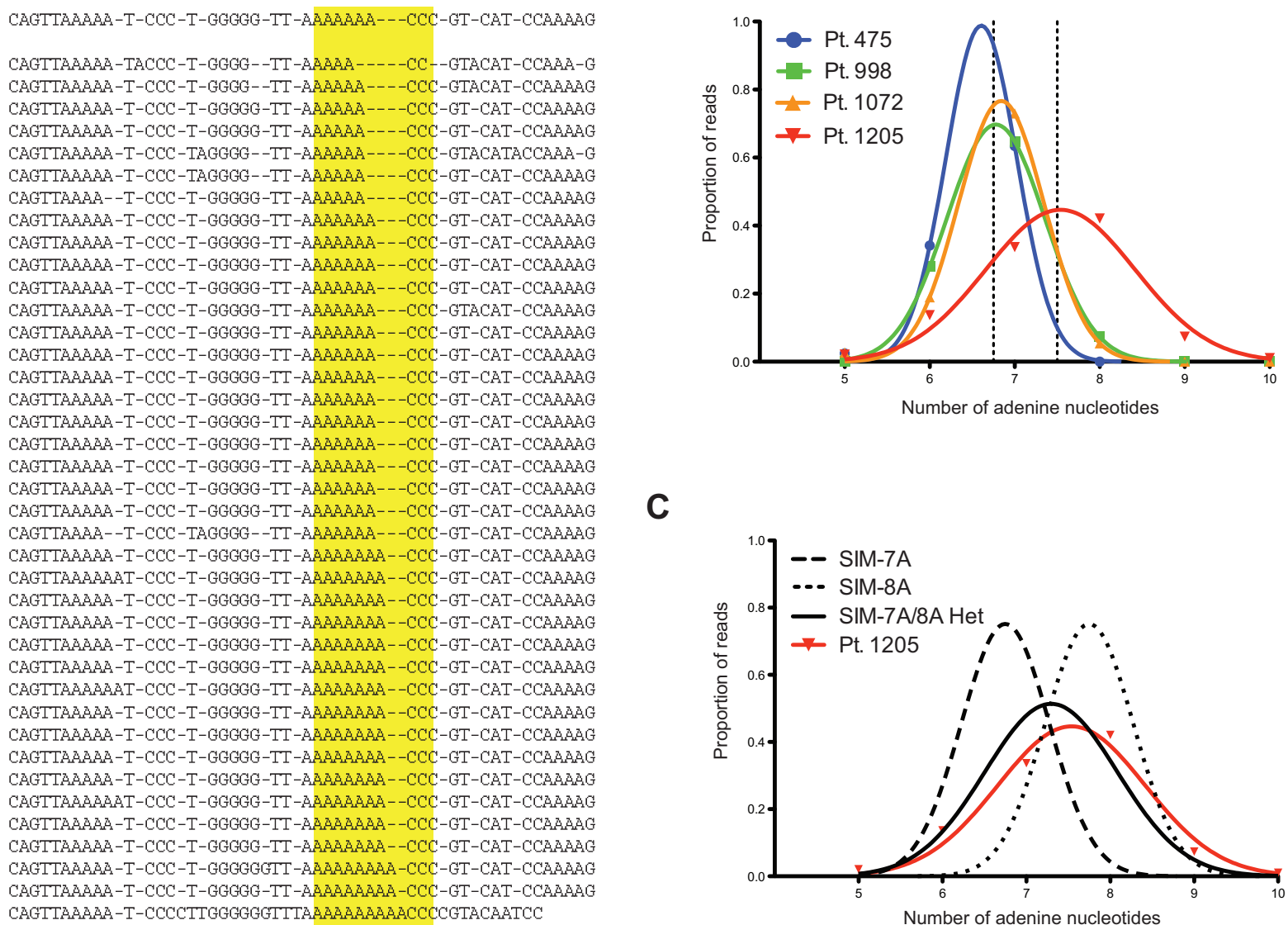

C

Fig. 2. Sequence analysis favors the existence of a heterozygous insertion in patient 1205. A, Local alignment of selected sequence reads from patient 1205 in the region containing a single-nucleotide insertion (hg18; chr5:13,754,404$13,754,447)$. The top line of the alignment represents the NCBI 36 reference sequence at this location. Gaps introduced into the alignment are represented by a "-." Yellow highlights the stretch of 7 adenine (7A) nucleotides where the insertion should result in 8 adenine ( $8 \mathrm{~A}$ ) nucleotides. $B$, The proportion of reads containing $5,6,7,8,9$, or 10 adenine nucleotides reveal a clear difference in patient 1205. Patients 475, 998, and 1072 act as "controls" as they are homozygous for reference 7A alleles. The highest proportions of reads in these samples were 7A, with Gaussian distributions $\left(R^{2}>0.998\right)$ having peaks at approximately $6.75(6.607,6.778$, and 6.842$)$, consistent with a homozygous 7A genotype at this position. In contrast, the number of adenine residues in the sample from patient 1205 ranged from 5 to 10 , with a Gaussian distribution $\left(R^{2}=0.964\right)$ that was broader than the first three patients and had a peak at 7.54. $C$, When compared with simulated genotypes, the distribution from patient 1205 most closely resembles the heterozygous distribution. The distributions of the three "control" samples were averaged to obtain a simulated homozygous 7A distribution (SIM-7A). This distribution was shifted to the right to generate a simulated homozygous 8A distribution (SIM-8A). The SIM-7A and SIM-8A distributions were averaged to simulate the expected distribution in an individual heterozygous for 7A and 8A (SIM-7A/8A Het).

substitution variants (Fig. 3A). Although there were no dramatic differences between the types of variants in terms of total number of sequence reads (Fig. 3B), higher read depth was associated with tighter clustering of "known" dbSNP variants near $50 \%$ or $100 \%$ of the reads (Figs. 3C and 3D), demonstrating the greater precision that can be achieved with higher depth of coverage. Similarly, novel substitution variants tended to cluster near $50 \%$ or $100 \%$ of the reads, with the exception of four variants observed in fewer than $15 \%$ of reads that were thus called homozygous wild type (Fig. 3E). In contrast, the putative novel insertion/deletion variants displayed a completely different pattern in which there was no direct relationship between the read depth and the percentage of reads containing the variant
(Fig. 3F). As noted earlier, Sanger sequencing did not confirm any of the eight putative novel insertion/deletion variants detected by 454 sequencing. Thus, the accuracy of 454 sequencing for detection of small insertion/deletion variants is not ideal, especially for novel insertion/deletion variants identified in fewer than $25 \%$ of sequence reads.

Because the four patient samples were previously subjected to extensive Sanger sequencing of PCD-associated genes (DNAH5, DNAH11, DNAI1, WDR63, RSPH3/RSHL2, RSPH4A/RSHL3, and RSPH6A/RSHL1), many benign sequence variants were known to be present in addition to the presumptive disease-causing mutations (see Table, Supplemental Digital Content 6, http://links.lww.com/GIM/A135). We used this ad- 
A

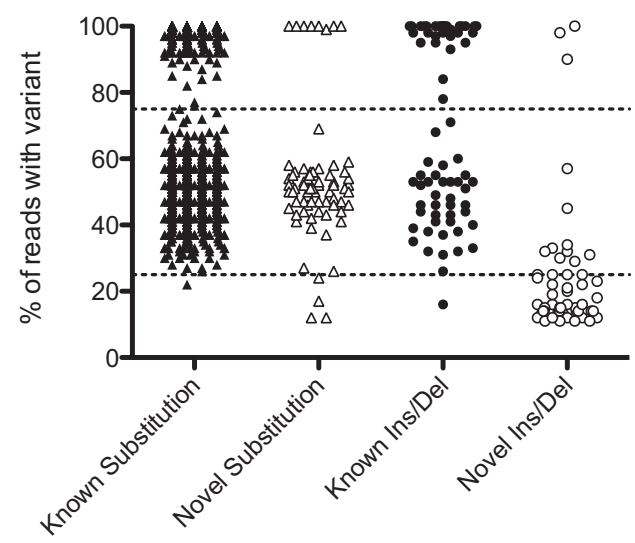

C

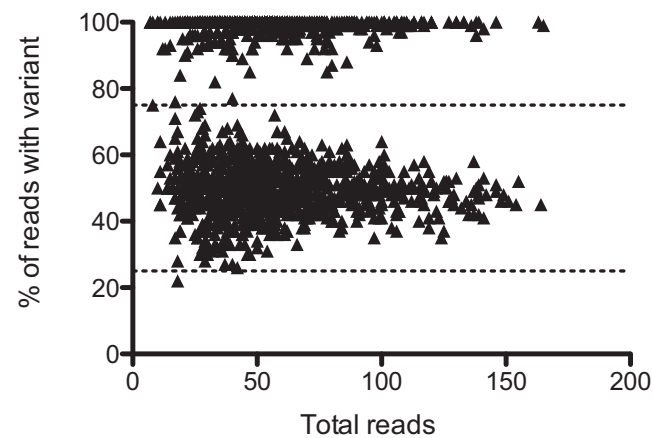

E

Novel Substitutions

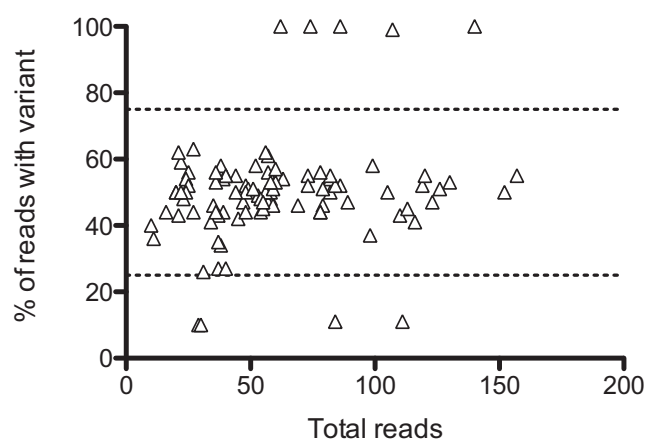

B

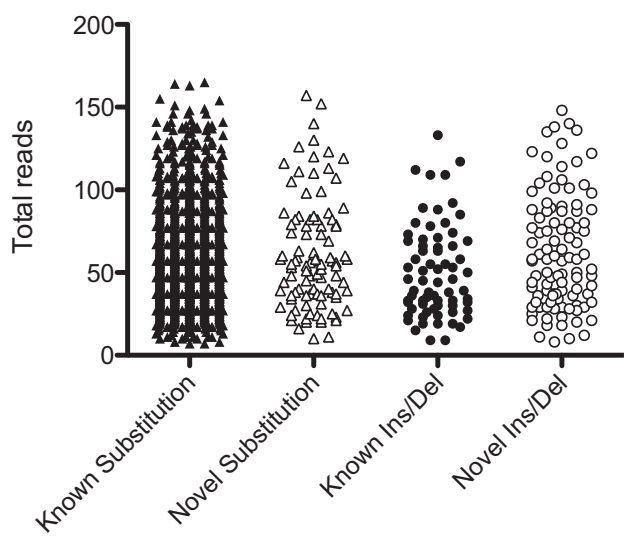

\section{Known Insertions/Deletions}

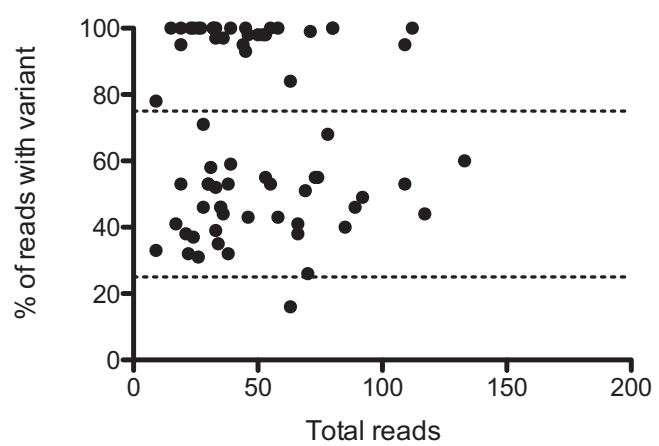

$\mathbf{F}$

\section{Novel Insertions/Deletions}

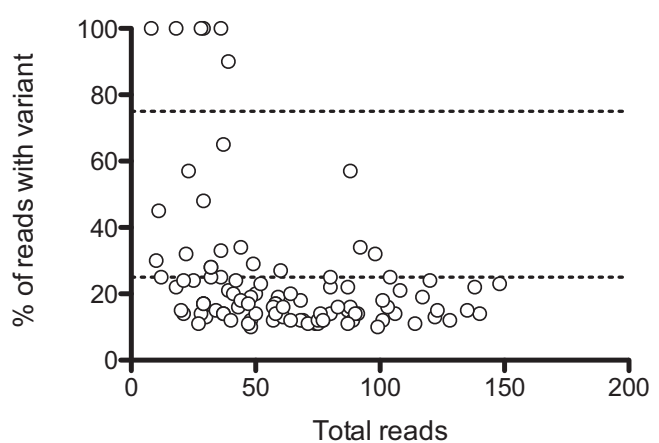

Fig. 3. Small insertion/deletion variants are observed in distinct proportions of sequence reads. A, Variants were separated by class and whether they were novel or previously reported in dbSNP and plotted according to the percentage of sequence reads in which the variant was observed. Dashed lines represent $25 \%$ and $75 \%$ of sequence reads. Substitution variants largely clustered near $50 \%$ or $100 \%$ of sequence reads as would be expected for heterozygous or homozygous variants, respectively. Known insertion/deletion variants (Ins/Del) also tended to cluster near 50\% or 100\% of sequence reads. However, novel small insertion/deletion variants had a much wider distribution with a significant proportion of variants detected in fewer than $25 \%$ of the sequence reads. B, The depth of coverage, defined by the number of sequence reads in the alignment for a given variant, is shown for the different types of sequence variants. No obvious differences were observed between the classes of variants in terms of the total depth of coverage. C-F, Known substitutions, known insertions/deletions, novel substitutions, and novel insertions/deletions were plotted according to the total read depth and the percentage of reads in which the variant was observed. The novel insertion/deletion variants clearly follow a distinct pattern, with a large proportion of the variants detected in fewer than $25 \%$ of sequence reads, but no clear relationship between depth of coverage and the percentage of reads in which the variant was observed. 
ditional information to analyze the sensitivity of our targeted exome massively parallel sequencing approach for detection of sequence variants. Of the 119 benign variants that had previously been found within the exon-targeted region, 114 were also identified with the expected zygosity using massively parallel sequencing. Four of the discrepancies (three single-base substitutions and one 2-bp deletion) were found to be homozygous wild type when reanalyzed by Sanger sequencing and, thus, were determined to be false-positive calls by the previous automated Sanger sequencing. The remaining variant was a complex alteration in the region of an oligo-dT tract near the end of an intron of WDR63 (hg18; chr1:85,346,294$85,346,301)$. Prior Sanger sequencing identified one allele with a single $\mathrm{C}$ insertion and another allele with a $\mathrm{CT}$ insertion, immediately preceding the oligo-dT tract. Massively parallel sequencing correctly detected the homozygous $\mathrm{C}$ insertion (rs11427716) but not the additional heterozygous $\mathrm{T}$ insertion. This result further demonstrates the difficulties posed by homopolymer tracts for 454 sequencing.

\section{DISCUSSION}

Targeted exome massively parallel sequencing has great potential for both research and clinical use. On the basis of the work by $\mathrm{Ng}$ et al. ${ }^{9}$ with whole-exome sequencing, each individual likely has 6-12 genes with two predicted damaging mutations that are not present in control populations (dbSNP, HapMap). In a clinically distinctive autosomal recessive disorder with only one disease locus, discovery of the disease gene by whole-exome sequencing could require as few as three or four unrelated individuals. ${ }^{7}$ However, because of the genetic heterogeneity of PCD (and many other genetic disorders), a much larger number of individuals might need to be analyzed to identify genes in common among subsets of such patients. We are currently engaged in whole-exome sequencing of additional patients with PCD whose mutations remain unidentified (M.A.Z. and M.R.K.). However, although the research implications of next-generation sequencing are clear, use of this emerging technology for clinical diagnostic testing still requires careful calibration.

\section{Detection of known mutations associated with PCD}

In this work, we conducted a pilot study of four patients with clinical features consistent with PCD and with previously identified mutations in genes associated with PCD. We specifically chose samples with qualitatively different types of mutations occurring in different genes to determine whether targeted exome capture combined with next-generation sequencing could identify different classes of mutations. The exome capture of 79 known or candidate PCD genes, followed by massively parallel sequencing using 454 technology and structured analysis, identified five of the seven known mutations associated with PCD in three of the four patients, including one patient (475) with two previously identified mutations in the dynein heavy-chain gene DNAH5 (a nucleotide substitution resulting in a splice site alteration and a 21-bp deletion) and another patient (1072) with two different single-nucleotide nonsense mutations in the dynein intermediate-chain gene DNAI1. In the third patient (998), previous analysis had identified only one disease-causing mutation in DNAH11 (a 4-bp frameshift deletion). This mutation was incorrectly excluded by the initial analytic scheme due to its annotation as a known SNP but was correctly identified after a simple adjustment of the handling of annotated SNPs. In the fourth patient (1205), the single-base frameshift insertion could only be demonstrated retrospectively with analysis of sequence reads, but the exonic deletion was not detected.

\section{Variants of uncertain clinical significance}

A number of other heterozygous and homozygous novel variants were identified in the four patients. Although these variants are of unknown clinical significance, it is tempting to speculate that some could play a role in the PCD phenotype, acting as genetic modifiers. Further massively parallel sequencing of a large number of patients with PCD with known deleterious mutations could lend further insight into the roles of putative disease-modifying variants.

The patient in whom a single disease-causing mutation in DNAH11 had previously been identified (patient 988) was found in this study to have a missense variant in DNAH2. Mutation profiling of DNAH2 has not been carried out in patients with PCD, but mutations of the Chlamydomonas ortholog (Dhc10) alter flagellar motility. ${ }^{33}$ The DNAH2 variant affected a highly conserved amino acid residue that was predicted to be damaging, and family segregation analysis was consistent with the possibility of digenic inheritance. Although this finding does not prove that digenic variants of DNAH11 and $D N A H 2$ can result in pathogenicity, digenic inheritance has been previously hypothesized in PCD, ${ }^{34}$ and there is precedent for triallelic inheritance in a different ciliopathy, Bardet-Biedel syndrome. ${ }^{35}$ It is worth noting that DNAH11 encodes an outer dynein arm protein, whereas $D N A H 2$ encodes an inner dynein arm protein; because both DNAH11 and DNAH2 proteins are components of the ciliary dynein arms, which require complex protein interaction for motility, it is thus possible that haploinsufficiency of these two different genes could result in reduced ciliary function without a structural defect discernable by electron microscopy. We speculate that this patient's phenotype could be due to the combinatorial effects of mutations in $D N A H 11$ and DNAH2. However, it is also possible that there simply remains an undetected mutation in a regulatory element, promoter, or intron of DNAH11 or DNAH2, or biallelic mutations in a gene not present among the 79 genes analyzed in this study.

Finally, it is worth noting in this context that massively parallel approaches such as whole-exome and whole-genome sequencing will result in the identification of thousands of protein coding variants in each individual, any of which could potentially impact disease (either directly as a disease-causing mutation or as a genetic modifier), rendering the determination of clinical significance exceedingly complex with respect to various combinations of mutations. For example, if alternative inheritance patterns such as digenic inheritance are considered, the number of different possibilities quickly becomes astronomical when variants are simultaneously detected in hundreds or thousands of genes. This will be a major challenge faced by molecular diagnosticians and clinicians in the future as multiplex gene sequencing is more broadly applied.

\section{Performance and limitations of targeted exome massively parallel sequencing}

In addition to the previously identified disease-causing mutations, we analyzed 119 benign variants previously identified in the four patients by automated Sanger sequencing. Of these, targeted exome massively parallel sequencing correctly identified 114 variants and revealed four of these putative variants to be false-positive results of the Sanger sequencing. Thus, the final 454 and Sanger sequencing results were in agreement for 114 of 115 benign polymorphic variants and $>99 \%$ of wild- 
type reference calls. Including the six previously identified small deleterious mutations, we calculate a sensitivity of $98.3 \%$ $(119 / 121)$ for detection of sequence variants by exon capture followed by 454 sequencing. The majority $(>90 \%)$ of the polymorphisms studied in this context were single-nucleotide substitution variants, so this result primarily confirms that 454 sequencing has high sensitivity and specificity for the detection of substitution mutations.

All the variants in which there was discordance between the final 454 and Sanger sequencing results were small insertions or deletions. This drawback is inconvenient but not devastating for researchers, who expect and tolerate a certain level of error, but it is of particular importance for clinical diagnostic testing. Small insertion and deletion mutations make up approximately $22 \%$ of the approximately 100,000 known mutations in the human gene mutation database (http:/www.hgmd.cf.ac.uk/), and inability to reliably identify this class of mutations would be a major limitation of adopting 454 sequencing for clinical diagnostic testing.

In an empirical study using a library of reference templates, Huse et al. ${ }^{31}$ found that insertions and deletions were the most common errors observed with 454 pyrosequencing, comprising $63 \%$ of all sequence errors. These calculations were based on individual reads, whereas variant detection is based on the percentage of mapped reads that contain the variant, so it is unclear how this error rate might translate directly to inaccurate detection of insertion/deletion variants. In our experience, the majority of putative novel (not previously reported in dbSNP) insertion/deletion variants were observed in fewer than $25 \%$ of the sequence reads, and this phenomenon seemed to be independent of the depth of coverage. Sanger sequencing did not confirm any of the eight putative novel single-nucleotide insertions that we analyzed.

One explanation for the detection of sequence variants in fewer than $25 \%$ of reads could be the existence of pseudogenes not currently annotated in the reference genome but nevertheless contributing to the material being sequenced (e.g., HYDIN; see Text and Figure, Supplemental Digital Content 7, http://links.lww.com/GIM/A136). However, in this case, one might expect recurrent substitution and insertion/deletion variants affecting the same few genes (those with previously undiscovered pseudogenes or paralogous copies) and for the same variants to be present in more than one individual. Neither of these was the case in our experience, suggesting that false-positive insertion/deletion identification is largely inherent to 454 sequencing technology. Thus, many (perhaps most) of the novel insertion/ deletion variants detected in $<25 \%$ of 454 sequence reads seem to be artifacts of the biochemistry, bioinformatics, or both. Restricting analysis only to variants detected in $>25 \%$ of sequence reads greatly improved the specificity of this approach without affecting sensitivity (data not shown).

In our study, a single-base insertion mutation in patient 1205 was only identified retrospectively by demonstrating that the sequence data were consistent with the presence of the heterozygous insertion in comparison with the three other patients. This false-negative result thus represents a combined platform and bioinformatics failure. Indeed, the 454 bioinformatics pipeline seems to use proprietary models to compensate for the imprecision of pyrosequencing with respect to homopolymers, perhaps explaining why the true deleterious insertion mutation in patient 1205 was not called by GS Reference Mapper but was called by NextGENe. As noted earlier, the increased sensitivity of the NextGENe analysis came at the unacceptable cost of lower apparent specificity, because a much higher number of putative small insertion/deletion variants were detected in the vicinity of homopolymer stretches. As all possibly deleterious variants will require confirmation with clinical Sanger sequencing, at least in the short term, follow-up sequencing could be very costly or impractical with a high falsepositive rate for novel insertion/deletion variants.

It was not entirely unexpected that the approach described in this study was unable to detect a whole-exon deletion in the DNAH5 gene in patient 1205 , because targeted exon capture is not designed to detect large deletions. We attempted to uncover evidence for the whole-exon deletion in structural variant alignments and analysis of sequence read depth but were unable to find such confirmation. Variability in the depth of coverage between exons and between samples in our study seems to have been too high to reliably detect changes in exon copy number using nonquantitative exon-capture methods. Interestingly, while this article was under review, another group published a report of a similar approach to detect inherited mutations for breast and ovarian cancer, in which deletions or duplications encompassing single or multiple exons were, in fact, accurately detected. ${ }^{36}$ Possible reasons for the success of Walsh et al. include differences in platforms or technical aspects of the exon-capture process, a $>10$-fold higher mean depth of coverage $(\sim 1200 \times)$, and a larger number of samples between which comparisons could be made.

\section{CONCLUSIONS}

Massively parallel sequencing has already proven successful in research settings, and its adoption for clinical diagnostic testing seems to be inevitable, particularly as the costs per base continue to decrease. Nevertheless, clinical tests based on nextgeneration sequencing present special challenges not germane to research applications and will need careful validation to assess the performance characteristics of this new technology and to optimize the analytical sensitivity and specificity. Nextgeneration sequencing platforms may have inherent differences in detection of diverse types of variants, ${ }^{37,38}$ so any clinical applications based on next-generation technologies will need to be validated on different types of known reference mutations to determine their performance characteristics. Analytic software for clinical diagnostic approaches will also need to be optimized for detection of different types of mutations. Reliance on dbSNP as a bioinformatics filter seems to be problematic, given that some rare deleterious mutations are contained among the many benign variants. We expect that as whole-exome and wholegenome sequencing are carried out in large numbers of patients and control subjects, an approach will evolve toward using information about the population frequency of a variant, rather than its presence or absence in a database, as a key filter for evaluating the likely clinical implications of a given variant. We conclude that exon capture followed by massively parallel sequencing has considerable potential in clinical diagnostics, but deficiencies will need to be quantified and rectified before widespread adoption in a clinical setting.

\section{ACKNOWLEDGMENTS}

This work was supported, in part, by grant M0O1RR00046 and UL-1-RR025747 from the National Center of Research resources, NIH. J.S.B. is supported by the University Cancer Research Fund (UCRF; http://ucrf.unc.edu/). J.P.E. is supported by the UNC Center for Genomics and Society (NHGRI 5-P50HG004488-03) and a UNC Clinical Translational Science Award (1-UL1-RR025747-01); M.R.K., M.A.Z., and M.W.L. are supported by NIH research grant 5-U54-HL096458-06, 
funded by the office of the Director, and supported by ORDR and NHLBI, NIH; M.R.K. and M.A.Z. are supported by NIH grant 5-R01HL071798; H.O. is supported by a grant of the "Deutsche Forschungsgemeinschaft" (DFG Om 6/4 to H.O.); and MLPA was supported by a Multidisciplinary Research Grant (MRG) to K.E.W. and M.A.Z., North Carolina Biotechnology Center. The authors are grateful to the patients and their families for their participation in this study. The authors thank the German patient support group "Kartagener Syndrom und Primaere Ciliaere Dyskinesie e.V." The authors appreciate technical assistance from L. Huang, A. Cutting, A. Heer, J. Wallmeier, Dr. H. Olbrich, and Dr. K. Chao.

\section{REFERENCES}

1. Noone PG, Leigh MW, Sannuti A, et al. Primary ciliary dyskinesia: diagnostic and phenotypic features. Am J Respir Crit Care Med 2004;169:459467.

2. Leigh MW, Pittman JE, Carson JL, et al. Clinical and genetic aspects of primary ciliary dyskinesia/Kartagener syndrome. Genet Med 2009;11:473487.

3. Kennedy MP, Omran H, Leigh MW, et al. Congenital heart disease and other heterotaxic defects in a large cohort of patients with primary ciliary dyskinesia. Circulation 2007;115:2814-2821.

4. Zariwala MA, Knowles MR, Leigh MW. Primary ciliary dyskinesia. In: Pagon RA, Bird TC, Dolan CR, Stephens K, editors. GeneReviews [Internet]. Seattle, WA: University of Washington, Seattle, 1993-2007 [updated October 6, 2009]. PMID: 20301301.

5. Hornef N, Olbrich H, Horvath J, et al. DNAH5 mutations are a common cause of primary ciliary dyskinesia with outer dynein arm defects. $A m J$ Respir Crit Care Med 2006;174:120-126.

6. Zariwala MA, Leigh MW, Ceppa F, et al. Mutations of DNAII in primary ciliary dyskinesia: evidence of founder effect in a common mutation. Am J Respir Crit Care Med 2006;174:858-866.

7. Ng SB, Turner EH, Robertson PD, et al. Targeted capture and massively parallel sequencing of 12 human exomes. Nature 2009;461:272-276.

8. Choi M, Scholl UI, Ji W, et al. Genetic diagnosis by whole exome capture and massively parallel DNA sequencing. Proc Natl Acad Sci USA 2009; 106:19096-19101.

9. $\mathrm{Ng} \mathrm{SB}$, Buckingham $\mathrm{KJ}$, Lee $\mathrm{C}$, et al. Exome sequencing identifies the cause of a mendelian disorder. Nat Genet 2010;42:30-35.

10. Lupski JR, Reid JG, Gonzaga-Jauregui C, et al. Whole-genome sequencing in a patient with Charcot-Marie-Tooth neuropathy. $N$ Engl J Med 2010;362: 1181-1191.

11. Sobreira NL, Cirulli ET, Avramopoulos D, et al. Whole-genome sequencing of a single proband together with linkage analysis identifies a Mendelian disease gene. PLoS Genet 2010;6:e1000991.

12. Biesecker LG. Exome sequencing makes medical genomics a reality. Nat Gen 2010;42:13-14.

13. ten Bosch JR, Grody WW. Keeping up with the next generation: massively parallel sequencing in clinical diagnostics. J Mol Diagn 2008;10:484-492.

14. Tucker T, Marra M, Friedman JM. Massively parallel sequencing: the next big thing in genetic medicine. Am J Hum Genet 2009;85:142-154.

15. Voelkerding KV, Dames SA, Durtschi JD. Next-generation sequencing: from basic research to diagnostics. Clin Chem 2009;55:641-658.

16. Vasta V, Ng SB, Turner EH, Shendure J, Hahn SH. Next generation sequence analysis for mitochondrial disorders. Genome Med 2009;1:100.

17. Ashley EA, Butte AJ, Wheeler MT, et al. Clinical assessment incorporating a personal genome. Lancet 2010;375:1525-1535.
18. Wheeler DA, Srinivasan M, Egholm M, et al. The complete genome of an individual by massively parallel DNA sequencing. Nature 2008;452:872-876.

19. Droege M, Hill B. The Genome Sequencer FLX ${ }^{\mathrm{TM}}$ system-longer reads, more applications, straight forward bioinformatics and more complete data sets. J Biotechnol 2008;136:3-10.

20. Sunyaev S, Ramensky V, Koch I, Lathe W 3rd, Kondrashov AS, Bork P. Prediction of deleterious human alleles. Hum Mol Genet 2001;10:591-597.

21. Ramensky V, Bork P, Sunyaev S. Human non-synonymous SNPs: server and survey. Nucleic Acids Res 2002;30:3894-3900.

22. Castleman VH, Romio L, Chodhari R, et al. Mutations in radial spoke head protein genes RSPH9 and RSPH4A cause primary ciliary dyskinesia with central-microtubular-pair abnormalities. Am J Hum Genet 2009;84:197-209.

23. Loges NT, Olbrich H, Becker-Heck A, et al. Deletions and point mutations of LRRC50 cause primary ciliary dyskinesia due to dynein arm defects. $\mathrm{Am} \mathrm{J}$ Hum Genet 2009;85:883-889.

24. Maiti AK, Mattéi MG, Jorissen M, Volz A, Zeigler A, Bouvagnet P. Identification, tissue specific expression, and chromosomal localisation of several human dynein heavy chain genes. Eur J Hum Genet 2000;8:923932

25. Schwabe GC, Hoffmann K, Loges NT, et al. Primary ciliary dyskinesia associated with normal axoneme ultrastructure is caused by DNAH11 mutations. Hum Mutat 2008;29:289-298

26. Bartoloni L, Blouin JL, Maiti AK, et al. Axonemal beta heavy chain dynein DNAH9: cDNA sequence, genomic structure, and investigation of its role in primary ciliary dyskinesia. Genomics 2001;72:21-33.

27. Sherry ST, Ward MH, Kholodov M, et al. dbSNP: the NCBI database of genetic variation. Nucleic Acids Res 2001;29:308-311.

28. Zariwala M, Noone PG, Sannuti A, et al. Germline mutations in an intermediate chain dynein cause primary ciliary dyskinesia. Am J Respir Cell Mol Biol 2001;25:577-583.

29. Zuccarello D, Ferlin A, Garolla A, et al. A possible association of a human tektin-t gene mutation (A229V) with isolated non-syndromic asthenozoospermia: case report. Hum Reprod 2008;23:996-1001.

30. Zhang Z, Jones BH, Tang W, et al. Dissecting the axoneme interactome: the mammalian orthologue of Chlamydomonas PF6 interacts with sperm-associated antigen 6, the mammalian orthologue of Chlamydomonas PF16. Mol Cell Proteomics 2005;4:914-923.

31. Huse SM, Huber JA, Morrison HG, Sogin ML, Welch DM. Accuracy and quality of massively parallel DNA pyrosequencing. Genome Biol 2007;8: R143.

32. Raca G, Jackson C, Warman B, Bair T, Schimmenti LA. Next generation sequencing in research and diagnostics of ocular birth defects. Mol Genet Metab 2010;100:184-192.

33. Perrone CA, Myster SH, Bower R, O'Toole ET, Porter ME. Insights into the structural organization of the I1 inner arm dynein from a domain analysis of the 1beta dynein heavy chain. Mol Biol Cell 2000;11:2297-2313.

34. Blouin J-L, Meeks M, Radhakrishna U, et al. Primary ciliary dyskinesia: a genome-wide linkage analysis reveals extensive locus heterogeneity. Eur $J$ Hum Genet 2000;8:109-118.

35. Katsanis N, Ansley SJ, Badano JL, et al. Triallelic inheritance in BardetBiedl syndrome, a Mendelian recessive disorder. Science 2001;293:22562259.

36. Walsh T, Lee MK, Casadei S, et al. Detection of inherited mutations for breast and ovarian cancer using genomic capture and massively parallel sequencing. Proc Natl Acad Sci USA 2010;107:12629-12633.

37. Dames S, Durtschi J, Geiersbach K, Stephens J, Voelkerding KV. Comparison of the Illumina Genome Analyzer and Roche 454 GS FLX for resequencing of hypertrophic cardiomyopathy-associated genes. J Biomol Tech 2010;21:73-80.

38. Hoppman-Chaney N, Peterson LM, Klee EW, Middha S, Courteau LK, Ferber MJ. Evaluation of oligonucleotide sequence capture arrays and comparison of next-generation sequencing platforms for use in molecular diagnostics. Clin Chem 2010;56:1297-1306. 